\title{
SER CUIDADOR DE FAMILIAR COM CÂNCER
}

\author{
CARING FOR FAMILY WITH CANCER
}

\section{SER CUIDADOR DE FAMILIAR CON CÁNCER}

\author{
Maria de Lourdes da Silva Marques Ferreira* \\ Maria EugÊNia Mutro** \\ Carla Regiani Conde ${ }^{* * *}$ \\ Maria José SANCHES MARIN ${ }^{* * *}$ \\ Silmara MENEguin ${ }^{* * * *}$ \\ Fernanda Moerbeck Cardoso MazZetTo ${ }^{* * * * *}$
}

\begin{abstract}
RESUMO
Objetivo: Compreender a experiência do cuidador familiar de paciente com câncer. Método: Estudo qualitativo, com abordajem fenomenológico, realizado no período de janeiro a junho de $2013 \mathrm{em}$ uma Unidade de Tratamento Oncológico, baseado em entrevista aberta com 14 cuidadores por meio da questão: Qual é a sua experiência em cuidar do (nome)? Resultados: Foram identificadas quatro categorias como resultantes da experiência do cuidador: representação do câncer na família, repercussões do câncer na vida pessoal do cuidador, enfrentando a repercussão da não aceitação da doença do familiar e a finitude, sendo cuidador com sentimentos de gratidão. Conclusão: Foi atribuído à função de cuidador mudança na rotina, com repercussões físicas, psicológicas e impotência. O cuidar foi visto também como satisfação pessoal, retribuição de cuidados aos pais. A experiência de ser cuidador traz sobrecarga e mudanças na estrutura familiar e a equipe de saúde pode oferecer apoio formal e atuar como facilitadora neste processo.
\end{abstract}

Palavras chave: Cuidador familiar, câncer, oncologia, enfermagem oncológica.

\begin{abstract}
Objective: To understand the experience of family caregivers of patients with cancer. Method: Qualitative study using a phenomenological approach, conducted from January to June 2013 in an Oncological Treatment Unit, based on open interviews with 14 caregivers through the question: "What is your experience in the care of (name)?” Results: Four categories were identified as a result of the caregiver experience: representation of cancer

“ Enfermeira. Docente do Departamento de Enfermagem, Universidade Estadual Paulista "Júlio de Mesquita Filho", Faculdade de Medicina de Botucatu, Botucatu, São Paulo, Brasil. Email: malusa@fmb.unesp.br. Autor correspondente.

${ }^{* *}$ Enfermeira. Mestre em Enfermagem pelo Programa de Pós-graduação Mestrado Profissional em Enfermagem, Universidade Estadual Paulista "Júlio de Mesquita Filho", Faculdade de Medicina de Botucatu, Botucatu, São Paulo, Brasil. Email:maromutro@fmb.unesp.br

${ }^{* * *}$ Enfermeira. Doutoranda pelo Programa de Pós-graduação em Enfermagem, Universidade Estadual Paulista "Júlio de Mesquita Filho”, Faculdade de Medicina de Botucatu, Botucatu, São Paulo, Brasil. Email: carlaregiani@yahoo.com.br

${ }_{* * * *}$ Enfermeira. Docente do Departamento de Enfermagem, Faculdade de Medicina de Marília, Marília, São Paulo, Brasil. Email:marnadia@terra.com.br

***** Enfermeira. Docente do Departamento de Enfermagem, Universidade Estadual Paulista "Júlio de Mesquita Filho", Faculdade de Medicina de Botucatu, Botucatu, São Paulo, Brasil. Email:silmeneguin@fmb.unesp.br

${ }^{* * * * * *}$ Enfermeira. Doutoranda pelo Programa de Pós-graduação em Enfermagem, Universidade Estadual Paulista "Júlio de Mesquita Filho”, Faculdade de Medicina de Botucatu, Botucatu, São Paulo, Brasil. Email: fmcmazzetto@hotmail.com
\end{abstract}


in the family, cancer repercussions on the caregiver's personal life, facing the repercussion of the denial of the family member's illness and finitude, and being a caregiver with feelings of gratitude. Conclusions: It was attributed to the role of caregiver a change in routine, with physical and psychological repercussions, and feelings of impotence. The care was also seen as personal satisfaction, and retribution of care to parents. The experience of being a caregiver brings a burden and changes in family structure and the health team can offer formal support and act as a facilitator in this process.

Key words: Family caregiver, cancer, oncology, oncology nursing.

\section{RESUMEN}

Objetivo: Comprender la experiencia del cuidador familiar de pacientes con cáncer. Método: Estudio cualitativo, con abordaje fenomenológico, realizado de enero a junio de 2013 en una Unidad de Tratamiento Oncológico, basado en entrevistas abiertas con 14 cuidadores a través de la pregunta: ¿Cuál es su experiencia en el cuidado de (nombre)? Resultados: Se identificaron cuatro categorías como resultado de la experiencia de ser cuidador: representación del cáncer en la familia, repercusiones del cáncer en la vida personal del cuidador, enfrentándose a la repercusión de la no aceptación de la enfermedad del familiar y la finitud, y ser cuidador con sentimientos de agradecimiento. Conclusión: Se atribuye a la función de cuidador cambios en la rutina, con efectos físicos, psicológicos e impotencia. La atención también se ve como la satisfacción personal, y retribución al cuidado de los padres. La experiencia de ser cuidador trae sobrecarga y cambios en la estructura familiar y el equipo de salud puede ofrecer apoyo formal y actuar como un facilitador en este proceso.

Palabras clave: Cuidador familiar, cáncer, oncología, enfermería oncológica.

Fecha recepción: 21/01/2017 Fecha aceptación: 29/04/2018

\section{INTRODUÇÃO}

As doenças crônicas, e dentre elas o câncer, são as principais causas de morte no mundo. Dessa forma, desde a década de 1970 a pessoa com câncer em fase avançada, juntamente com seus familiares ou amigos, denominados cuidadores informais, são considerados pelos serviços de saúde como um conjunto a ser cuidado. Neste contexto, os cuidadores formam uma variável significativa para a assistência, cabendo-lhes a tarefa de prestar o cuidado cotidiano ao seu ente querido (1).

A elevada incidência de doenças crônico-degenerativas na população de idosos é característica da transição epidemiológica mundial desde o último século (2). No Brasil, as doenças crônicas constituem o problema de saúde de maior magnitude, sendo cerca de $70 \%$ das causas de morte (3). A Organização
Mundial da Saúde considera o atendimento às necessidades dos cuidadores objetivo central dos cuidados paliativos e determina que se disponibilize sistemas de apoio para ajudar a família. Tal rede de suporte, portanto, não apenas é um agente facilitador de cuidados continuados, mas um importante apoio para cuidadores, quando devidamente articulado com diferentes serviços de saúde (4).

Atualmente, o sistema de cuidado à saúde tem proposto mudanças no modo de assistir o indivíduo com doenças crônicas avançadas e incentivado o cuidado domiciliar. Essa alteração no local de prestação do cuidado faz com que aumente a responsabilidade familiar, e, por sua vez, a família pode não estar preparada para assumir o doente, necessitando ser informada sobre a doença, tratamento, e receber instrução sobre habilidades técnicas para o cuidado. Assim, a rede e apoio social são recursos que a equipe de enferma- 
gem pode oferecer para melhoria da qualidade de vida dessas famílias (5), pois cuidar de paciente com doença crônica ou avançada no domicílio pode gerar sobrecarga de trabalho considerável aos cuidadores familiares, que também podem adoecer, por ficar com a maior parte das tarefas na assistência ao paciente (6).

A ansiedade, medo da morte, incerteza da cura e melhora do estado de saúde do enfermo, são sentimentos constantes no cenário do diagnóstico e tratamento do câncer, principalmente em fase terminal. Associados a estes sentimentos, altos índices de mortalidade e medo da morte tornam-se constante, e a família é fonte importante de apoio para a mulher, que deve participar ativamente no enfrentamento destas dificuldades, levandoa a ter sentimentos de coragem e esperança. Por outro lado, a repercussão da doença na vida do paciente, associado com efeitos colaterais do tratamento, acaba tendo consequências importantes para a família, principalmente para o cuidador principal (7).

Dessa forma, justifica-se a necessidade de conhecer as experiências dos cuidadores de pacientes com câncer, a fim de facilitar a organização da rede de cuidados tanto por parte dos familiares como dos serviços de saúde que orientam a assistência ao doente e cuidador. Tal prática é importante para subsidiar as estratégias de intervenções visando atender as necessidades físicas e psicossociais no ambiente familiar. O câncer é uma doença associada ao sofrimento do indivíduo e familiar e o cuidado cotidiano, mesmo que informal, prestado pelo familiar é exaustivo fisicamente e psicologicamente, necessitando de suporte emocional. Sendo assim, a questão norteadora foi: "Fala para mim, ¿qual a sua experiência em cuidar do senhor/senhora? (nome da pessoa)" e, na tentativa de responder este questionamento, o estudo apresentou como objetivo compreender a experiência do cuidador familiar do paciente com câncer.

\section{MATERIAIS E MÉTODO}

O fenômeno ser cuidador de familiar foi experiência vivida por um dos autores do estudo que, embora sua experiência não foi exatamente com um doente com diagnóstico de câncer, a significação foi de sofrimento para ambos y de a necessidade de uma reestruturação familiar, visando alívio da dor vivida pelos cuidadores e pela pessoa a ser cuidada.

Esse estudo também se reporta ao familiar cuidador e trata-se de estudo descritivo de abordagem qualitativa com referencial metodológico a fenomenologia.

A fenomenologia é a modalidade do fenômeno situado, cuja essencialidade refere-se à compreensão abrangente e profunda dos dados. A pesquisa fenomenológica propõe "ir à coisa mesma", ou seja, àqueles que possuem experiência do fenômeno interrogado em seu mundo-vida, e por isto, podem falar sobre ele (8). Busca-se, desta forma, essa essência e significação da realidade vivenciada pelos cuidadores familiares que atuam como cuidadores principais de pacientes com câncer.

O fenômeno se mostra quando situado, é interrogado na região de inquérito que nesta pesquisa, foi a experiência do cuidador familiar de pacientes com câncer em acompanhamento em Hospital Estadual de Bauru (HEB), interior do estado de São Paulo, Brasil.

Foi desenvolvido no período de janeiro a junho de 2013, unidade de internação/ambulatório oncológico do HEB e contou com a participação de 14 cuidadores de pacientes portadores de câncer. Incluíram na seleção cuidadores que faziam parte da família do paciente, com idade superior a 18 anos e que eram considerados o cuidador principal. Os participantes foram abordados na sala de espera do ambulatório e na internação oncológica enquanto aguardava o familiar. Nesse momento, esclareceu-se a natureza da pesquisa, objetivos, métodos e implicações, 
dando-lhes o direito da escolha de participar.

Considerando os critérios consolidados para questões da pesquisa qualitativa (9), os pesquisadores deixaram claros como ia ser conduzida a pesquisa para a coleta de dados, identificação dos pesquisadores, relação estabelecida com os participantes, desenho do estudo, o tamanho da amostragem, o registro da entrevista, a duração da mesma e a clareza dos temas resultantes.

O tamanho da amostragem do estudo foi definido pela saturação dos dados (10), e todos os cuidadores convidados a participar da pesquisa aceitaram, e ao receber o aceite, a pesquisadora responsável pela coleta dos dados, os encaminhava a uma sala reservada. Essa pesquisadora atuava como enfermeira no ambulatório de oncologia e tinha uma familiaridade com os participantes, o que, possibilitou coletar os depoimentos de forma espontânea e sem constrangimentos.

Cada participante primeiramente lia e assinava o termo de consentimento livre e esclarecido (TCLE) em conformidade com os aspectos éticos para pesquisas em seres humanos, segundo a Resolução CNS no 466/12. O projeto foi aprovado pelo comitê de Ética em Pesquisa da Faculdade de Medicina de Botucatu, por meio do ofício no 75/11-CEP.

Para a coleta de dados, optou-se pela entrevista aberta para que o entrevistado pudesse expressar-se livremente. Quando se analisa esse tipo de fonte, é preciso incorporar o contexto de sua produção e, se possível, complementar com informações da observação participante (11). As entrevistas foram registradas em um gravador eletrônico e posteriormente transcritas, analisadas e apagadas. A duração da entrevista teve média de 30 a 40 minutos com transcrição na finalização de cada uma e direcionada questão norteadora, deixando os cuidadores à vontade para expressar sua experiência com familiar com câncer.

No que tange ao rigor científico do estudo qualitativo o processo de pesquisa precisa ter valor próprio, aplicabilidade, consistência e neutralidade por forma a ter valor científico e isto independentemente de se admitir que a natureza do conhecimento dentro do paradigma racionalista ou quantitativo seja diferente do conhecimento obtido na pesquisa dentro do paradigma interpretativo (12).

As variáveis para a caracterização sociodemográfica foram: idade, estado civil, renda familiar, grau de parentesco entre cuidador e paciente e tempo de cuidado prestado ao familiar doente.

Com os dados coletados em mãos, os pesquisadores, autores deste artigo, iniciaram a análise dos depoimentos, começando pelas transcrições das falas e percorreram os três momentos descritos no referencial fenomenológico: descrição, redução e compreensão ou interpretação dos depoimentos (8). Mais tarde, situado o fenômeno e recolhidas as descrições, iniciou-se a análise idiográfica e nomotética pautado na linha filosófica do fenômeno situado de Husserl (13).

As categorias temáticas foram derivadas dos dados, apresentadas nos resultados e os participantes tiveram um feedback das descobertas e puderam sanar dúvidas o que possibilitou o atendimento de necessidades das famílias.

A análise ideográfica buscou tornar visível a ideologia presente na descrição ingênua, o pesquisador busca unidades de significado, após várias leituras de cada descrição. Essas leituras prévias aproximaram os pesquisadores ao fenômeno, e familiarização com o conteúdo das descrições. As unidades de significados foram recortes considerados significativos pelos pesquisadores diante da interrogação do estudo (11).

A análise nomotética foi realizada com base na análise das divergências e convergências expressas pelas unidades de significado, vinculada a interpretações que os pesquisadores fizeram. Assim, novos grupos foram formados e, no processo de convergências e interpretações, surgiram novas categorias. Essas generalidades iluminaram uma nova perspectiva do fenômeno (14). 
Inicialmente, os pesquisadores realizaram repetidas leituras atentivas dos discursos, visando apreender o sentido do todo e, na sequência, identificaram-se as unidades de significado, caminhando em direção à intersubjetividade, chegando-se à descrição das evidências da experiência vivida, destacando-se os dados significativos para compreensão do fenômeno pesquisado, que foram destacados e enumerados sequencialmente (15).

A extração da essencialidade da experiência do cuidador por meio da identificação das unidades de significados possibilitou a construção das categorias, levou a uma redução na busca da "vivência do familiar cuidador", partindo da interrogação da pesquisa. Após, estabeleceu-se a redução fenomenológica, cujas expressões cotidianas dos sujeitos da pesquisa foram transformadas na linguagem do pesquisador (16).

Em seguida foi realizada etapa de compreensão, iniciando-se pela análise ideográfica que se refere à individualidade de cada discurso, de forma a evidenciar a estrutura do fenômeno situado: ser cuidador de familiar com câncer, atendidos na unidade de internação/ambulatório oncológico do HEB, em busca das unidades de significados, agrupadas pela categoria comum, com objetivo de organizar as articulações do discurso conforme suas significações sobre essa vivência (16).

Após, procedeu-se a análise nomotética, análise geral dos discursos. Nesta análise buscou-se a generalidade para apreender aspectos mais comuns dos depoimentos. Inicialmente foram agrupados temas que emergiram das unidades interpretadas por meio da redução fenomenológica (8).

Para garantir o anonimato dos cuidadores, primeiramente foi estabelecido um código "Dep" para cada participante é, respeitando a ordem da realização das entrevistas, o primeiro entrevistado foi identificado por "Dep 1", o segundo "Dep 2" e assim sucessivamente.

Norteada pelo objetivo proposto no pre- sente estudo, a análise fenomenológica das entrevistas revelou que, ao relatarem a experiência, os participantes descreveram toda sua história e vivências.

\section{RESULTADOS}

Neste espaço apresentam-se os dados sociodemográficos dos participantes e, em seguida, as categorias de análise.

Dados sociodemográficos dos cuidadores: Participaram do estudo 14 cuidadores de familiares com câncer com idades entre 32 a 60 anos. A maioria era casada (64,3\%). Quanto ao grau de parentesco entre cuidador e paciente, a maioria era filhos $(64,3 \%)$. A renda familiar teve predomínio de 1 a 2,5 salários e a maioria referiu que cuidou do familiar por um período de 1 a 5 anos, e apenas um referiu 11 anos.

Categoria 1. Representação do câncer na família. Essa categoria define o significado ao câncer diante da experiência de cuidar de familiar e sentimentos de fraternidade. Contou, ainda, com a incerteza se o cuidado prestado estava sendo realizado corretamente devido a que situação de adoecimento do familiar ser algo novo:

Cuidar de paciente oncológico representa um aprendizado, pois exige conhecimento por parte do cuidador no que se refere às formas de cuidar e de equilíbrio (Dep 13). Eu nunca tinha cuidado de uma pessoa doente, do jeito que ele está... com câncer. É muito duro de aceitar tudo isto.... Até porque é pai da gente nesta situação, eu fico muito comovida... (Dep 8). Fiquei em choque quando soube, é duro para aceitar (Dep 7). Eu sinto que o cuidador tem que apresentar equilíbrio para poder ajudar (Dep 5).

Categoria 2. Repercussão do câncer na vida pessoal do cuidador. Expressa sentimentos do cuidador no que se referem às funções al- 
teradas, bem como emoções desencadeadas pelo fato de tomar conta de um familiar, a sobrecarga, dificuldade em conciliar a vida pessoal com o papel de cuidador, restrições em sair de casa, de descanso e de sono:

Pra mim está difícil, porque eu tenho filha pré adolescente, tenho que dar atenção, e minha mãe que é uma idosa que está com um problema de saúde sério (Dep 1). Não é fácil (...) porque eu não aguento com ele, não tenho quem me ajude. Me dói as costas (Dep 2). Antes, quando ela andava, a gente dava banho de chuveiro $e$ trocava, agora é na cama, a gente não sabe, fica nervosa (Dep 6). Ah quase não saio de casa, eu cuido dele, né?. Desde o começo. Eu cuido dele, então não posso viajar, eu nem penso (Dep 9).

\section{Categoria 3. Enfrentar a repercussão da não aceitação da doença pelo familiar e a finitu- de $e$ as dificuldades de cuidar da pessoa no domicílio. Essa categoria revela o significado da impotência do familiar diante da não acei- tação da doença pelo paciente e exprimem a percepção da finitude:}

Ele chora bastante, quer sair, trabalhar, a gente sabe que não pode, explica pra ele que não pode mais, corta o coração (Dep 12). É duro nessa situação dele, agora, pior ainda, até porque os médicos disseram que não tem mais o que fazer, é só esperar... (Dep 10). .... É muito difícil porque ...minha casa é simples, humilde, não tem conforto para dar pra ela..., não é ambiente ideal para ela (Dep 4). Eu nunca pedi nada para ninguém, mas eu me vi assim, numa situação tão difícil, tive que pedir para um para outro, você me ajuda com isso, tudo é difícil... (Dep 14).

\section{Categoria 4. Ser cuidador com sentimentos} de gratidão. Essa categoria exprime sentimentos de família, de gratidão pelos cuidados recebidos pelos pais e de inversão de papéis:

Pra mim a experiência de cuidar é tudo, é meu pai e eu gosto de ajudar (Dep 12). Minha experiência? Ah acho que é uma retribuição por tudo que ela fez por mim, e nesta minha experiência vejo como aprendizado é que a família se une... (Dep 11). Ele voltou a ser uma criança, e eu a sua mãe... cuido com o maior carinho (Dep 3).

\section{DISCUSSÃO E CONCLUSÃO}

Pacientes com câncer e suas famílias ao vivenciarem a confirmação do diagnóstico de neoplasia, o indivíduo portador da doença sente o desejo de ser cuidado, amado, compreendido e, principalmente, de poder compartilhar suas preocupações e medos. Nesses momentos, a família passa a enfrentar conflito emocional tendo que apoiar o ente querido e, ao mesmo tempo, aceitar o câncer. Diante desse contexto, se não considerar os sentimentos dos familiares no momento do diagnóstico e tratamento do câncer, principalmente dos cuidadores, a ajuda não tem como ser eficaz. O nosso estudo corrobora com a pesquisa de Souza y Gomes (17), que considera a necessidade dos familiares cuidadores apresentarem equilíbrio de seus próprios sentimentos, lidando com questões práticas da vida, na cotidianidade da doença. Neste sentido, um estudo realizado no México sobre a experiência de ser cuidador em doenças crônicas, refere que uma das principais fontes de estresse para este cuidador é a percepção dos sintomas do paciente (18), o que evidencia a necessidade de compreensão da doença do familiar que está sob seus cuidados.

Após o diagnóstico de uma doença crônica como o câncer, inicia-se o tratamento, que pode ser, ao mesmo tempo, incerto, doloroso e prolongado. Então, modifica-se o pensamento do paciente e de seus familiares e o percurso terapêutico pode deixar marcas profundas, pode chocar a família, podendo até afastar os amigos e provocar sentimentos e emoções de desespero por viver com uma 
doença estigmatizante (19). Assim, durante o enfrentamento inicial do câncer, as representações dos cuidadores deste estudo, no que se refere cuidar de um familiar com câncer, vêm de encontro aos sentimentos vivenciados no estudo de Encarnação y Farinasso (20). A pesquisa relaciona o câncer ao choque, desespero, depressão e à angústia diante do câncer. Esses sentimentos unidos geram a sensação de impotência, comumente enfrentada por meio da negação, isto é, uma busca de respostas inversas à realidade.

Em estudo sobre paciente idoso com câncer e cuidador familiar os autores descreveram a importância de escutar histórias de vida de pacientes e cuidadores familiares, a fim de compreender as estratégias de enfrentamento. Isso pode resultar em cuidado centrado no paciente-família mais adequado (21).

O câncer não altera apenas a saúde do paciente, também traz repercussões à saúde do cuidador, que passa a preocupar-se em excesso, deixa de cuidar-se, tem ansiedade e se sobrecarrega entre várias atividades diárias, deixando de realizar atividades de recreação e lazer (22). Em nosso estudo os depoimentos dos cuidadores trazem essas repercussões de sobrecarga emocional, física, social e de depressão. Mudanças na rotina e cuidar de um doente em fim de vida requer muita habilidade para lidar com a situação de doença, mas acima de tudo, atender a sobrecarga envolvida na realização do cuidado (23).

Neste sentido, Garassini (24) encontrou que normalmente uma só pessoa acompanha o paciente o tempo todo, e que este, de forma auto atribuída, assume esse papel. Os familiares acreditam que protagonizam um rol muito importante ao cuidar de seu ente querido, porém sofrem esgotamento e expressam o sentimento de estarem só e que necessitam de ajuda. A experiência de ser cuidador tem trabalhos em horas que poderia ser de descanso, e que o pós-diagnóstico, no primeiro ano, mostra comprometimento no bem-estar e qualidade de vida, e declina ao longo do resto da trajetória da doença (25).

Quanto ao enfrentamento de familiares, pesquisa revela que os cuidadores possuem necessidade de alteração nas atividades diárias. Quanto aos pacientes em tratamento de radio e quimioterapia se sentem muito cansados e enfraquecidos, deixam de fazer atividades como antes e por sua vez, os familiares compartilham desse momento difícil (26).

Muitos cuidadores não se desapegam da vida antiga que levavam até mesmo por ter outros membros da família com demandas diferentes, e ao mesmo tempo, não conseguem dar conta da vida atual. Entretanto, observa-se uma anulação das vontades pessoais do cuidador; este passa á viver exclusivamente os desejos do doente (18). Neste estudo foi verificado a anulação decorrente do cuidador estar só nesta tarefa.

Ao presenciar de perto as dificuldades e incertezas após o diagnóstico, a família reconhece-as como uma verdadeira batalha, com identificação da própria fragilidade. No que se refere ao enfrentamento, quer seja focado no problema quanto na emoção, normalmente é utilizado desde o diagnóstico até o tratamento. Reconhecidamente o apoio social ajuda a enfrentar as situações geradoras de sofrimento físico ou psicológico, como ocorre no adoecimento por câncer (27). Nesse sentido, Garassini (28) identifica estratégias para o enfrentamento desses conflitos, tais como: a aprendizagem, a perseverança, valorização da vida, fortalecimento da fé, o cuidado da saúde, a importância de cuidar dos outros serem bons, o apoio da família, conhecimento adquirido, como também, o compartilhamento com outras pessoas que vivem a mesma realidade.

Neste estudo, essas necessidades ficam evidenciadas quando os cuidadores levam o familiar para casa. O cuidador familiar necessita estar preparado para o enfrentamento desta situação e os profissionais de saúde, e em especial os de enfermagem, são fundamentais para que os cuidadores, diante das dificuldades evidenciadas no cotidiano, se 
sintam assistidos, informados e apoiados no desempenho do cuidado (29).

Nesse sentido, mesmo a família ficando abalada com a notícia, ela tem um papel importante na recuperação do familiar com câncer (30). É sabido que as emoções negativas, o medo e a ira constituem-se emoções normais e esperadas diante do diagnóstico do câncer e que os seres humanos mostram a capacidade de aceitar novas situações que implicam perdas e que mobilizam seus recursos e fortalezas para enfrentá-las (24).

Neste contexto, a família tem a missão de fortalecer o papel social do seu ente querido e não deixá-lo cultivar sentimentos como fraqueza e isolamento. Por conseguinte, a rede social é fundamental no processo de reabilitação e busca superação e enfrentamento do diagnóstico do câncer por parte do paciente e familiar. Frente a isso, a construção de uma rede de apoio pode ser uma forma de ser solidário e aclamar as inquietudes no momento da descoberta da doença $(31,32)$.

O processo de vivenciar uma doença grave traz momentos de reflexão aos familiares e doentes, pois o câncer está intimamente associado à finitude da vida. A possibilidade de morte produz desconforto, sensação de abandono e aniquilamento e, ao vivenciar o processo de morte do outro, os familiares também pensam na própria finitude. Assim, os membros envolvidos iniciam nova etapa da existência, vivenciando cada momento com intensidade, vivendo cada dia como se fosse o último, encarando a existência humana de forma saudável e verdadeira (33).

Portanto, o câncer, além de acarretar sofrimento, traz à tona a mortalidade humana que se tenta mascarar. Quando há uma situação da doença ocorre rompimento das atividades cotidianas, rotineiras, e, ao mesmo tempo, apresenta-se a possibilidade para olhar a si mesmo e entender que a vida se aproxima da finitude, se faz mais presente (33).

Para o familiar cuidador a percepção de que a vida do seu ente querido pode estar chegando ao fim lhe proporciona, principalmente, sentimento de "impotência". O cuidador, na tentativa de superar a perda e amenizar seu sofrimento, antecipa muitas vezes seu luto e procura recordar bons momentos vividos entre ele e o doente. Ainda, busca cuidar do adoentado de forma prazerosa, atribui à doença um significado de transformação para alcançar uma vida melhor e apoia-se em Deus, como forma de consolo e conforto espiritual (34).

Em contrapartida, ser cuidador de paciente com câncer também pode referir-se a experiência de sentimentos positivos, evidenciados pelos cuidadores, tais como zelo, carinho e gratificação, mesmo que o trabalho seja dispendioso em vários níveis - emocional, físico e financeiro (35).

Diante de todas as necessidades expressadas nos depoimentos dos cuidadores de familiares com câncer, é inegável o papel fundamental que o enfermeiro desempenha para oferecer o apoio a estes cuidadores. A relação e o intercâmbio com os profissionais de saúde, principalmente a equipe de enfermagem que disponibiliza maior tempo com o paciente, assim como com os familiares, possibilita o atendimento das necessidades apresentadas.

Desde as primeiras fases do processo do cuidado, já é demonstrada a necessidade de acompanhamento do paciente e seus familiares, oferecendo informações sobre a doença, o tratamento, as emoções resultantes deste quadro, a importância do cuidado compartilhado, a comunicação com a pessoa doente e sua família, bem como as adaptações às novas rotinas, podem se converter em elementos essênciais para o cuidado do paciente e seu cuidador (28).

O objetivo desta investigação foi alcançado, pois compreendeu-se que ser cuidador de familiar com câncer traz sobrecarga e mudanças significativas na estrutura da vida familiar, principalmente, diante do des- 
preparo trazendo ansiedade, insegurança e desgaste que geram sofrimento. $\mathrm{O}$ cuidador revelou repercussões físicas e psicológicas de sofrimento, necessidades de apoio social e impacto financeiro. Houve ainda a sensação de impotência para enfrentamento da doença e finitude. Por outro lado, sentimento de gratidão, por poder cuidar dos pais, reconhecendo a inversão de papéis entre pais e filhos.

Para ampliar a perspectiva do cuidado à família torna-se necessário compreender mudanças ocorridas na interação e funcionamento familiar. Dados da literatura, e este estudo permite destacar exigências e demandas enfrentadas por cuidadores, indicando estratégias favoráveis a ser consideradas no processo de tratamento.

A necessidade de ressignificação da própria vida se deve partir do próprio cuidador, mas, deve contar com a equipe de saúde, oferecendo apoio formal, não permitindo a condição de cuidador inseguro, solitário e adoecido pelo cansaço. Os cuidadores experienciam desafios físicos e psicossociais que merecem maior exploração para ajudá-los na concepção de novas intervenções.

Acredita-se que os resultados deste estudo contribuam para redirecionar as ações de enfermagem ao paciente com câncer e sua família, possibilitando ao cuidador maiores chances de proporcionar maior qualidade de vida ao seu familiar doente.

Além disso, a pesquisa oferece subsídios aos profissionais de saúde para que estratégias de cuidado sejam direcionadas a ações pautadas em uma prática assistencial considerando a unidade, paciente e família, que muitas vezes passam despercebidas. Essas ações trariam ao doente e seu cuidador familiar à possibilidade de um cuidado integral e contínuo, tendo como enfoque uma assistência humanizada atendendo as reais necessidades da pessoa com câncer e para o cuidador maior tranquilidade ao prestar cuidados.

Os limites deste estudo referem-se ao desenho da pesquisa, que trata de uma explora- ção profunda dos dados, por ser qualitativa, no entanto, não se pode chegar à generalização dos dados. Assim outros estudos com novas abordagens metodológicas podem colaborar para explorar as repercussões do câncer na família, e na assistência de cuidadores de familiar com câncer.

Por outro lado, as virtudes deste trabalho com abordagem qualitativa, aberto a possibilidade de encontrar realidades múltiplas, permitiram descrever os processos vivenciais dos familiares cuidadores dos pacientes oncológicos desde uma perspectiva de perdas e ganhos tanto pessoais quanto grupais.

\section{REFERÊNCIAS}

1. World Health Organization. Global Status Report on non-communicable diseases 2010 [Internet]. Geneva: World Health Organization; 2011 [ citado 20 jan 2017]. 162 p. Disponível em: http://www. who.int/nmh/publications/ncd_report_ full_en.pdf

2. Ministério da Saúde (BR). Plano de ações estratégicas para o enfrentamento das doenças crônicas não transmissíveis (DCNT) no Brasil - 2011-2020 [Internet]. Brasília: Ministério da Saúde; 2011 [citado 20 jan 2017]. 154 p. Disponível em: http://bvsms.saude.gov.br/bvs/publicacoes/plano_acoes_enfrent_dcnt_2011. pdf

3. Melo RMC, Rua MS, Santos CSVB. Necessidades do cuidador familiar no cuidado à pessoa dependente: uma revisão integrativa da literatura. Revista de Enfermagem Referência. 2014; ser IV (2): 143-51.

4. Silva EF, Paniz VMV, Laste V, Torres ILS. Prevalência de morbidades e sintomas em idosos: um estudo comparativo entre zonas rural e urbana. Cien Saude Colet. 2013; 18(4): 1029-40. 
5. Martín JM, Olano-Lizarraga M, Saracíbar-Razquin M. The experience of family caregivers caring for a terminal patient at home: A research review. Int J Nurs Stud. 2016; 64:1-12.

6. Queiroz AHAB, Pontes RJS, Souza AMA, Rodrigues TB. Percepção de familiares e profissionais de saúde sobre os cuidados no final da vida no âmbito da atenção primária à saúde. Cien Saude Colet. 2013; 18(9): 2615-23.

7. Santos IDL, Alvares RB, Lima NM, Mattias SR, Cestari MEW, Pinto KRTF. Câncer de mama: o apoio recebido no enfrentamento da doença. Journal of nursing UFPE on line [Internet]. 2017 [citado 30 abr 2018]; 11(Supl. 8): 3222-7. Disponível em: https://periodicos.ufpe. br/revistas/revistaenfermagem/article/ view/110187/22070

8. Kunz E. Esporte: Uma abordagem com a fenomenologia. Movimento [Internet]. 2000 [citado 20 jan 2017]; 6(12): I - XIII. Disponível em: https://www.ludopedio. com.br/v2/content/uploads/295225 Vol.\%206,\%20No\%2012\%20(2000).pdf

9. Booth A, Hannes K, Harden A, Noyes J, Harris J, Tong A. COREQ. Consolidated Criteria for Reporting Qualitative Studies. In Moher D, Altman DG, Schulz KF, Simera I, Wager E, eds. Guidelines for reporting health research: a user's manual. Oxford: John Wiley \& Sons Ltd; 2014. p. 214-26.

10. Holloway I, Wheller S. Phenomenology. In: Holloway I, Wheeler S, eds. Qualitative research in Nursing and Healthcare. 3rd ed. Oxford: Wiley-Blackwell; 2015. $368 \mathrm{p}$.

11. Maziero VG, Bernardes A, Spiri WC, Gabriel CS. Construindo significados sobre gerência da assistência: um estudo fenomenológico. Cienc Cuid Saude. 2014; 13(3): 563-70.

12. Guba EG, Lincoln YS. Do inquiry paradigms imply inquiry methodologies? In:
Fetterman DM, ed. Qualitative approaches to evaluation in education: The silent scientific revolution. London: Praeger; 1988. p. 89-115.

13. Husserl E. Conferências de Paris. 2 ed. Lisboa: Edições 70; 2017. 48 p.

14. Minayo MCS. O desafio do conhecimento: pesquisa qualitativa em saúde. $14^{\mathrm{a}} \mathrm{Ed}$. São Paulo: Hucitec; 2014. 416 p.

15. Roehe MV. Uma abordagem fenomenológico-existencial para a questão do conhecimento em psicologia. Estudos de Psicologia [Internet]. 2006 [citado 20 jan 2017]; 11(2):153-8. Disponível em: http://www.scielo.br/pdf/epsic/v11n2/ a04v11n2.pdf

16. Martins J, Bicudo MAV. A pesquisa qualitativa em psicologia: fundamentos e recursos básicos. 5 ed. São Paulo: Moraes; 2005. $110 \mathrm{p}$.

17. Souza MCG, Gomes AMT. Sentimentos compartilhados por familiares de pacientes oncológicos em tratamento quimioterápico: um estudo de representações sociais. Rev Enferm UERJ. 2012; 20(2): 149-54.

18. Aldana G, García L. La experiencia de ser cuidadora de un anciano con enfermedad crónica. Aquichan. 2011; 11(2): 158-72.

19. Silva PLN, Ruas PR, Barbosa HA, Soares LM, Rocha GG. O significado do câncer: percepção de pacientes. Journal of nursing UFPE on line [Internet]. 2013 [citado 20 jan 2017]; 7(12): 6828-33. Disponível em: https://periodicos.ufpe. $\mathrm{br} /$ revistas/revistaenfermagem/article/ view/12345/15064

20. Encarnação JF, Farinasso ALC. A família e o familiar cuidador de pacientes fora de possibilidades terapêuticas: uma revisão integrativa. Semina: Ciências Biológicas e da Saúde. 2014; 35(1): 137-48.

21. Senden C, Vandecasteele T, Vandenberghe E, Versluys K, Piers R, Grypdonck M, et al. The interaction between lived experiences of older patients and their family 
caregivers confronted with a cancer diagnosis and treatment: a qualitative study. Int J Nurs Stud. 2015; 52(1): 197-206.

22. Medeiros EGMS, Leite RFB, Ramos DKR, Almeida LAL. Repercussões do câncer infantil no cotidiano do familiar cuidador. Rev RENE. 2014; 15(2): 233-9.

23. Delalibera M, Presa J, Barbosa A, Leal I. Sobrecarga no cuidar e suas repercussões nos cuidadores de pacientes em fim de vida: revisão sistemática da literatura. Cien Saude Colet. 2015; 20(9): 2731-47.

24. Garassini MH. Los cuidadores de pacientes con cáncer: aprendizajes y recomendaciones a las familias, sociedades y centros de salud. Psicología y Salud. 2016; 26(2): 161-75.

25. Petricone-Westwood D, Lebel S. Being a caregiver to patients with ovarian cancer: A scoping review of the literature Gynecol Oncol. 2016; 143(1): 184-92.

26. Tomaz LA, Veras Junior EF, Carvalho PMG. Enfrentamento e resiliência de pacientes com câncer submetidos a tratamento quimioterapico. R. Interd. 2015; 8(2): 195-201.

27. Rodrigues JSM, Ferreira NMLA. Estrutura e funcionalidade da rede de apoio social do adulto com câncer. Acta Paul Enferm. 2012; 25(5): 781-7.

28. Garassini MH. Narrativas de familiares de pacientes con câncer. CES Psicol. [Internet]. 2015 [citado 30 abr 2018]; 8(2): 76-102. Disponível em: http://www.scielo.org.co/pdf/cesp/v8n2/v8n2a05.pdf

29. Teston EF, Santos AL, Cecilio HPM, Ma- noel MF, Marcon SS. A vivência de doentes crônicos e familiares frente a necessidade de cuidado. Cienc Cuid Saude. 2013; 12(1): 131-8.

30. Ribeiro VC, Portella SDC, Malheiro ES. Mulheres de meia idade e o enfrentamento do câncer de mama. Rev Cuid. 2014; 5(2): 799-805.

31. Silva SED, Cunha JO, Marques-Neto AC, Costa JG, Trindade FA, Fonseca ALG, et al. As representações sociais do câncer de mama e no colo do útero no conhecimento da enfermagem brasileira. Revista Eletrônica Gestão \& Saúde [Internet]. 2013 [citado 20 jan 2017]; 4(3): 1130-45. Disponível em: http://periodicos.unb.br/ index.php/rgs/article/view/14177/10104

32. Moraes MAA, Saleh AK, Martins ACP, Silva CP, Dourado FS, Silva LM. Processo saúde doença das mulheres com câncer cérvico uterino nas redes de atenção. Revista da Universidade Vale do Rio Verde. 2016; 14(1): 355-65.

33. Menossi MJ, Zorzo JCC, Lima RAG. A dialógica vida/morte no cuidado do adolescente com câncer. Rev Lat Am Enfermagem. 2012; 20(1): 126-34.

34. Magalhaes SB, Franco ALS. Experiência de profissionais e familiares de pacientes em cuidados paliativos. Arq Bras Psicol. 2012; 64(3): 94-109.

35. Fratezi FR, Gutierrez BAO. Cuidador familiar do idoso em cuidados paliativos: o processo de morrer no domicílio. Cien Saude Colet. 2011; 16(7):3241-8. 